\title{
Sitimagene Ceradenovec
}

National Cancer Institute

\section{Source}

National Cancer Institute. Sitimagene Ceradenovec. NCI Thesaurus. Code C162553.

A replication-deficient adenovirus type 5 (Ad5) with E1 and partial E3 deletions containing cDNA for the herpes simplex virus thymidine kinase (HSV-Tk), which, when administered in combination with ganciclovir (GCV), possesses potential antineoplastic activity. Following administration, transgene-expressing cells produce thymidine kinase, which phosphorylates GCV to ganciclovir triphosphate, a cytotoxic nucleotide analog that is incorporated into DNA resulting in chain termination and induction of apoptosis in rapidly dividing cells. This process spares normal neurons as they do not proliferate and are therefore not susceptible to the toxic effects of GCV metabolites. 